\title{
Quantum Lower Bound for Graph Collision Implies Lower Bound for Triangle Detection
}

\author{
Kaspars BALODIS ${ }^{1,2}$, Jānis IRAIDS ${ }^{1}$ \\ ${ }^{1}$ Faculty of Computing, University of Latvia, Rainga bulvāris 19, Riga, LV-1586, Latvia \\ 2 Institute of Mathematics and Computer Science, University of Latvia, Rainga bulvāris 29, \\ Riga, LV-1459, Latvia \\ balodis.kaspars@lu.lv, janis.iraids@gmail.com
}

\begin{abstract}
We show that an improvement to the best known quantum lower bound for GRAPHCOLLISION problem implies an improvement to the best known lower bound for TRIANGLE problem in the quantum query complexity model. In GRAPH-COLLISION we are given free access to a graph $(V, E)$ and access to a function $f: V \rightarrow\{0,1\}$ as a black box. We are asked to determine if there exist $(u, v) \in E$, such that $f(u)=f(v)=1$. In TRIANGLE we have a black box access to an adjacency matrix of a graph and we have to determine if the graph contains a triangle. For both of these problems the known lower bounds are trivial $(\Omega(\sqrt{n})$ and $\Omega(n)$, respectively) and there is no known matching upper bound.
\end{abstract}

Keywords: quantum query complexity, triangle detection, graph collision

\section{Introduction}

By $Q(f)$ we denote the bounded-error quantum query complexity of a function $f$. We consider the quantum query complexity for some graph problems.

Definition 1. In TRIANGLE problem it is asked whether an $n$-vertex graph $G=(V, E)$ contains a triangle, i.e. a complete subgraph on three vertices. The adjacency matrix of the graph is given in a black box which can be queried by asking if $(x, y) \in E$.

Recently there have been several improvements in the algorithms for the TRIANGLE problem in the quantum black box model. The problem was first considered by Buhrman et al. (2005) who gave an $O(n+\sqrt{n m})$ algorithm where $n$ is the number of vertices and $m$ - the number of edges. Later in 2007 Magniez et al. gave an $\tilde{O}\left(n^{13 / 10}\right)$ algorithm based on quantum walks. Introducing a novel concept - learning graphs, and using a new technique Belovs (2012b) was able to reduce the complexity to $O\left(n^{35 / 27}\right)$. Lee, Magniez, and Santha (2013) using a more refined learning graph approach reduced 
the complexity to $\tilde{O}\left(n^{9 / 7}\right)$. Currently the best known algorithm is by Le Gall (2014) who exhibited a quantum algorithm which solves the TRIANGLE problem with query complexity $\tilde{O}\left(n^{5 / 4}\right)$. Classically the query complexity of TRIANGLE is $\Theta\left(n^{2}\right)$; however, it is an open question whether TRIANGLE can be computed in time better than $O\left(n^{\omega}\right)$ where $\omega$ is the matrix multiplication constant.

Definition 2. In GRAPH-COLLISION $G$ problem a known $n$-vertex undirected graph $G=(V, E)$ is given and a coloring function $f: V \rightarrow\{0,1\}$ whose values can be obtained by querying the black box for the value of $f(x)$ of a given $x \in V$. We say that a vertex $x \in V$ is marked iff $f(x)=1$. The value of the GRAPHCOLLISION $_{G}$ instance is 1 iff there exists an edge whose both vertices are marked, i.e. $\exists(x, y) \in E f(x)=f(y)=1$.

By $Q$ (GRAPH-ColLisION) we mean the complexity of solving GraPH-COLLI$\operatorname{SION}_{G}$ for the hardest $n$-vertex graph $G$.

There has been an increased interest in the quantum query complexity of the GRAPH-COLLISION problem, mainly because algorithms for solving GRAPH-COLLISION are used as a subroutine in algorithms for the TRIANGLE problem by Magniez, Santha, and Szegedy (2007) and Boolean matrix multiplication by Jeffery, Kothari, and Magniez (2012).

The best known quantum algorithm for GRAPH-COLLISION for an arbitrary $n$ vertex graph has complexity $O\left(n^{2 / 3}\right)$ by Magniez, Santha, and Szegedy (2007). However, for some graph classes there are algorithms with complexity $O(\sqrt{n})$ (by Ambainis et al. (2013), Belovs (2012a), Gavinsky and Ito (2012), and Jeffery, Kothari, and Magniez (2012)). It is an open question whether for every $n$-vertex graph $G$ GRAPHCOLLISION $_{G}$ can be solved with $O(\sqrt{n})$ queries.

Contrary to the improvements in the algorithms for these two problems, the best known lower bounds for $Q$ (GRAPH-COLLISION) and $Q$ (TRIANGLE) are still the trivial $\Omega(\sqrt{n})$ and $\Omega(n)$ respectively, which follow from the reduction to search problem. Nonetheless these lower bounds seem hard to improve with the current techniques.

As mentioned before, algorithms for GRAPH-COLLISION have been used as a subroutine for constructing algorithms for the TRIANGLE problem, therefore an improved algorithm for GRAPH-COLLISION would result in an improved algorithm for TRIANGLE. In this paper we show a reduction in the opposite direction-that an improvement in the lower bound on $Q$ (GRAPH-COLLISION) would imply an improvement in the lower bound on $Q$ (TRIANGLE).

\section{Result}

Theorem 1. If there is a graph $G=(V, E)$ with $n$ vertices such that GRAPHCOLLISION $_{G}$ has quantum query complexity $t$ then TRIANGLE problem has quantum query complexity at least $\Omega(t \sqrt{n})$.

Proof. We show how to transform the graph $G$ into a graph $G^{\prime}$ with $3 n$ vertices so that it is hard to decide if $G^{\prime}$ contains a triangle. More precisely, we construct the graph $G^{\prime}$ 
in such a way that solving the TRIANGLE problem on $G^{\prime}$ is equivalent to solving $O R$ function from the results of $n$ independent instances of GRAPH-COLLISION $G$.

First, we want to get rid of any triangles in $G$, therefore we transform $G$ into an equivalent bipartite graph $G_{2}=\left(V_{2}, E_{2}\right)$ with $2 n$ vertices by setting $V_{2}=\left\{v_{1}, v_{2}\right.$ $v \in V\}$ and $E_{2}=\left\{\left(x_{1}, y_{2}\right) \mid(x, y) \in E\right\}$. The graph $G_{2}$ is equivalent to $G$ in the following sense-if we mark the vertices $v_{1}$ and $v_{2}$ in $G_{2}$ for every marked vertex $v$ in $G$, then $G_{2}$ has a collision iff $G$ has a collision. However, the graph $G_{2}$ does not contain any triangle (since it is bipartite).

Next, we add $n$ isolated vertices $z_{1}, \ldots, z_{n}$ to $G_{2}$ thereby obtaining a graph $G^{\prime}$. Let $f_{1}, \ldots, f_{n}: V \rightarrow\{0,1\}$ be the colorings from $n$ independent GRAPH-COLLISION G $_{G}$ instances. We add the edges $\left(z_{i}, v_{1}\right)$ and $\left(z_{i}, v_{2}\right)$ to $G^{\prime}$ iff $v \in V$ is marked by the respective coloring, i.e., iff $f_{i}(v)=1$.

See Fig. 1 for an example.
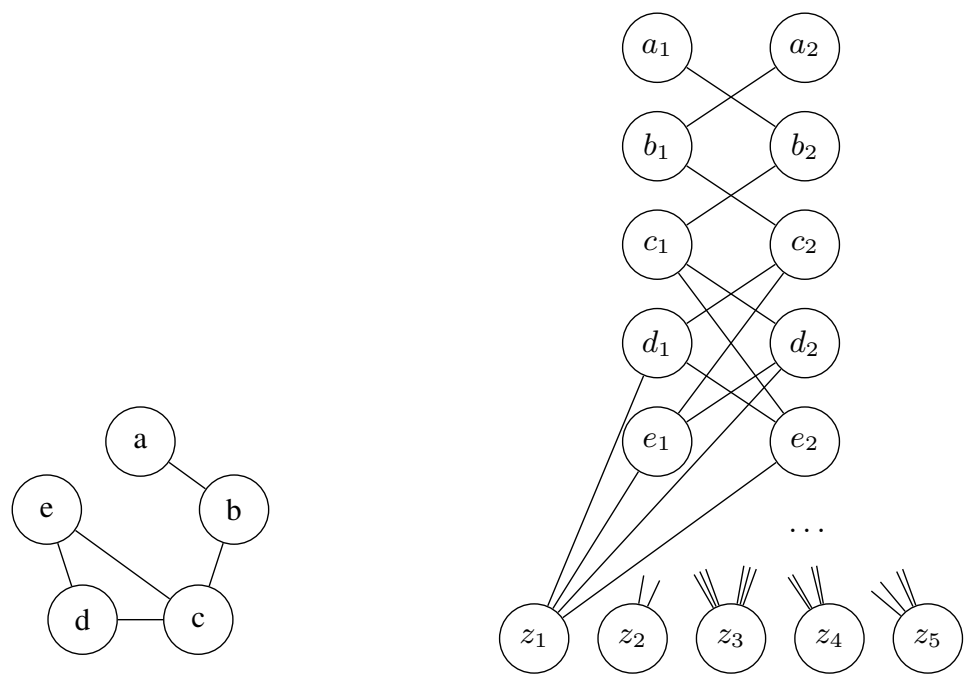

Fig. 1. Graph $G$ and the resulting graph $G^{\prime}$

The only possible triangles in the graph $G^{\prime}$ can be of the form $\left\{z_{i}, v_{1}, w_{2}\right\}$ for some $i \in\{1, \ldots, n\}$ and $v, w \in V$. Moreover, there is a triangle $\left\{z_{i}, v_{1}, w_{2}\right\}$ iff $f_{i}$ is such coloring that $G$ has a collision $(v, w)$, i.e., iff $f_{i}(v)=f_{i}(w)=1$. Therefore detecting a triangle in $G^{\prime}$ is essentially calculating $O R$ function from the results of $n$ instances of GRAPH-COLLISION $_{G}$.

We now use the fact that $O R$ function requires $\Omega(\sqrt{n})$ queries, the assumption that GRAPH-COLLISION $_{G}$ requires $t$ queries and the Theorem 1.5. from Reichardt (2011):

Theorem 2. Let $f:\{0,1\}^{n} \rightarrow\{0,1\}$ and $g:\{0,1\}^{m} \rightarrow\{0,1\}$. Then

$$
Q(f \bullet g)=\Theta(Q(f) Q(g)),
$$

where $(f \bullet g)(x)=f\left(g\left(x_{1}, \ldots, x_{m}\right), \ldots, g\left(x_{(n-1) m+1}, \ldots, x_{n m}\right)\right)$. 
Setting $f=O R$ and $g=$ GRAPH-COLLISION $_{G}$ gives the desired bound.

As the next corollary shows, a better lower bound on GRAPH-COLLISION implies a better lower bound on the TRIANGLE problem.

Corollary 1. If $Q_{2}($ GRAPH-COLLISION $)=\omega(\sqrt{n})$ then $Q_{2}($ Triangle $)=\omega(n)$.

\section{Acknowledgments}

The research leading to these results has received funding from the European Union Seventh Framework Programme (FP7/2007-2013) under grant agreement $n^{\circ} 600700$ (QALGO), ERC Advanced Grant MQC, Latvian State Research programme NexIT project No.1, and the project 271/2012 from the Latvian Council of Science.

\section{References}

Ambainis, Andris et al. (2013). "Parameterized quantum query complexity of graph collision". In: Proceedings of Workshop on Quantum and Classical Complexity. arXiv preprint: http: //arxiv.org/abs/1305.1021 pp. 5-16.

Belovs, Aleksandrs (2012a). "Learning-graph-based quantum algorithm for k-distinctness". In: IEEE 53rd Annual Symposium on Foundations of Computer Science (FOCS). arXiv preprint: http://arxiv .org/abs/1205.1534 IEEE, pp. 207-216. URL: http://ieeexplore. ieee.org/xpls/abs_all.jsp?arnumber $=6375298$.

Belovs, Aleksandrs (2012b). "Span programs for functions with constant-sized 1-certificates: extended abstract". In: Proceedings of the Forty-fourth Annual ACM Symposium on Theory of Computing. STOC '12. arXiv preprint: http://arxiv.org/abs/1105.4024. New York, New York, USA: ACM, pp. 77-84. ISBN: 978-1-4503-1245-5. DOI: 10 . 1145/2213977. 2213985 URL: http://doi.acm.org/10.1145/2213977.2213985

Buhrman, Harry et al. (2005). "Quantum algorithms for element distinctness". In: SIAM Journal on Computing 34.6. arXiv preprint: http : / / arxiv . org / abs / quant - ph / 0007016 pp. 1324-1330. DOI: 10 . 1137 / S0097539702402780 eprint: http : / / dx . doi . org / 10 . 1137 / S0097539702402780 URL: http : / / dx . doi . org / 10 . 1137 / S0097539702402780

Le Gall, François (2014). "Improved quantum algorithm for triangle finding via combinatorial arguments". In: IEEE 55rd Annual Symposium on Foundations of Computer Science (FOCS). arXiv preprint: http://arxiv.org/abs/1407.0085 pp. 216-225. DOI:10.1109/FOCS. 2014.31

Gavinsky, Dmitry and Tsuyoshi Ito (2012). "A quantum query algorithm for the graph collision problem”. In: arXiv e-prints. http://arxiv.org/abs/1204.1527 URL: http://arxiv . org/abs/1204.1527

Jeffery, Stacey, Robin Kothari, and Frédéric Magniez (2012). "Improving quantum query complexity of Boolean matrix multiplication using graph collision”. In: Automata, Languages, and Programming. arXiv preprint: http : / / arxiv . org/abs /1112 . 5855. Springer, pp. 522-532. URL: http : //link . springer . com/chapter/10 . 1007/978-3-64231594-7_44

Lee, Troy, Frédéric Magniez, and Miklos Santha (2013). "Improved quantum query algorithms for triangle finding and associativity testing". In: Proceedings of the Twenty-Fourth Annual ACM-SIAM Symposium on Discrete Algorithms. arXiv preprint: http://arxiv.org/abs/ 1210.1014 SIAM, pp. 1486-1502. 
Magniez, Frédéric, Miklos Santha, and Mario Szegedy (2007). "Quantum algorithms for the triangle problem". In: SIAM Journal on Computing 37.2. arXiv preprint:http://arxiv.org/ abs/quant-ph/0310134 pp. 413-424. URL: http://epubs.siam.org/doi/abs/10. $1137 / 050643684$

Reichardt, Ben W. (2011). "Reflections for quantum query algorithms". In: Proceedings of the Twenty-Second Annual ACM-SIAM Symposium on Discrete Algorithms. SODA '11. arXiv preprint: http://arxiv.org/abs/1005.1601. San Francisco, California: SIAM, pp. 560569. URL: http://dl.acm.org/citation. cfm?id=2133036.2133080

Received August 22, 2016, accepted September 16, 2016 